\title{
Second-Order Statistics of Complex Signals
}

\author{
Bernard Picinbono, Fellow, IEEE, and Pascal Bondon, Member, IEEE
}

\begin{abstract}
The second-order statistical properties of complex signals are usually characterized by the covariance function. However, this is not sufficient for a complete second-order description, and it is necessary to introduce another moment called the relation function. Its properties, and especially the conditions that it must satisfy, are analyzed both for stationary and nonstationary signals. This leads to a new perspective concerning the concept of complex white noise as well as the modeling of any signal as the output of a linear system driven by a white noise. Finally, this is applied to complex autoregressive signals, and it is shown that the classical prediction problem must be reformulated when the relation function is taken into consideration.
\end{abstract}

\section{INTRODUCTION}

C OMPLEX SIGNALS are used in various areas of signal processing. In the continuous-time (CT) case, they appear, for example, in the description of narrowband signals. Indeed, the appropriate definition of instantaneous phase or amplitude of such signals requires the introduction of the socalled analytic signal, which is necessarily complex [1]. In the discrete-time (DT) case, the parametric representation of signals is not restricted to real signals [2]. In fact, ARMA signals can very well be complex either because the coefficients of the rational function used in the transfer function are complex or because the driving white noise is complex. Finally, sampling of CT complex signals introduces complex DT signals. This paper is mainly restricted to the DT case, even though many results can be transferred to the CT case without any difficulty.

Let $z[k]$ be a complex DT signal, where $k$ is an integer describing the time dependence. Being interested in statistics concerning $z[k]$, we shall assume that it is random and that its expectation is zero. This last assumption simplifies the mathematical processing with no loss of generality. The second-order statistics of $z[k]$ are usually described by the covariance function $(\mathrm{CF})$ defined by

$$
\gamma_{z}\left[k_{1}, k_{2}\right]=E\left\{z\left[k_{1}\right] z^{*}\left[k_{2}\right]\right\}
$$

where the star indicates the complex conjugate. However, this $\mathrm{CF}$ is not sufficient to entirely describe the second-order statistics of $z[k]$. For this purpose, one is obliged to introduce another function, called, for simplicity, the relation function (RF), and defined by

$$
r_{z}\left[k_{1}, k_{2}\right]=E\left\{z\left[k_{1}\right] z\left[k_{2}\right]\right\} .
$$

Manuscript received September 17, 1995; revised August 5, 1996. The associate editor coordinating the review of this paper and approving it for publication was Dr. Ananthram Swami.

The authors are with the Laboratoire des Signaux et Systèmes, CNRS (UMR 14), École Supérieure d'Électricité, 91192 Gif-sur-Yvette, France.

Publisher Item Identifier S 1053-587X(97)01181-1.
In various instances, this RF is equal to zero and can, therefore, be omitted. This is the case for the analytical signal of any stationary signal (see [1 p. 230]) and, more generally, for any circular signal [3]. Indeed, a second-order circular signal is defined by the property $r_{z}\left[k_{1}, k_{2}\right]=0$. The term of circularity comes from the following remark. It is clear from (1) that the CF's of $z[k]$ and of $\exp (j \alpha) z[k]$ are equal for any real number $\alpha$. Now, the RF's of $z[k]$ and of $\exp (j \alpha) z[k]$ are equal if and only if $r_{z}\left[k_{1}, k_{2}\right]=0$. A second-order circular signal is a signal whose second-order statistics are invariant in any phase transformation. Hence, the relation $r_{z}\left[k_{1}, k_{2}\right]=0$ characterizes the circularity of $z[k]$. As an obvious consequence, real signals cannot be circular. However, the RF has generally no reason to be equal to zero, and therefore, it is necessary to describe the second-order statistics of a random signal completely. Complex signals are introduced in many textbooks, especially in [4], which is entirely devoted to properties of complex stochastic processes that are not trivial extensions of those of real processes. However, it is worth pointing out that the RF has never been introduced and analyzed. This is one of the purposes of this paper.

More precisely, our aim is to study various properties of the pair of correlation and relation functions. In particular, it is well known that the $\mathrm{CF}$ is not an arbitrary function because it must be nonnegative definite. Conversely, any nonnegative definite function is a CF. Then, the class of $\mathrm{CF}$ 's and the class of nonnegative definite functions are the same. In the same perspective, the RF cannot be arbitrary, and this is analyzed in Section II. More precisely, we establish a necessary condition that must be satisfied by $r_{z}\left[k_{1}, k_{2}\right]$ or its Fourier transform in order to be an RF. Various consequences of this condition are analyzed in Section III. In order to show that the condition introduced in Section II is sufficient, it is appropriate to solve the modeling (or inverse) problem. It consists of showing that to any pair of $\mathrm{CF}$ and RF satisfying the previous condition, it is possible to associate at least one complex random signal having these functions as CF or RF. A classical way for this purpose is a modeling procedure in which the random signal is generated by filtering a white noise. However, the concept of complex white noise must be clarified. Indeed, whiteness is a property concerning only the CF. Then, there exits a degree of freedom on the RF, which permits us to introduce many kinds of white noises, extending some results known for real signals [5], [6]. As in the real case, the modeling problem is much more complex than the direct one and has no unique solution. Indeed, it is easy to see that many different complex signals can have the same second-order statistics. Various procedures of modeling are 
analyzed in Section IV, and this introduces new perspectives on the concepts of AR signals that are analyzed in Section V.

\section{CONDitions ON SECOND-ORdER Statistics}

\section{A. The Concepts of Stationarity}

A complex signal $z[k]$ is said to be wide sense stationary (WSS) if its mean value is constant and if its CF (1) is only a function of $k_{1}-k_{2}$ (see [2, p. 51], [7, p. 95], [8, p. 15], [9, p. 120], or [10, p. 66], among others). This definition does not imply any condition on the RF (2).

We shall say that a signal is second-order stationary (SOS) if it is WSS and if its RF is also only depending on $k_{1}-k_{2}$. It is clear that for real signals, the two concepts are equivalent. On the other hand, for complex signals, WS stationarity does not imply SO stationarity, and some examples of this fact will be presented in Section III.

\section{B. Second-Order Stationary Signals}

The assumption of SO stationarity means that the CF and the RF of $z[k]$ are only depending on the difference $k_{1}-k_{2}$. Let us also assume that they have Fourier transforms denoted $\Gamma_{z}(\nu)$ and $R_{z}(\nu)$, respectively. The problem we want to discuss is to exhibit some necessary properties that these functions must satisfy. If $z[k]$ is real, these functions are equal, and $\Gamma_{z}(\nu)$ is the power spectrum of the signal. It is known that $\Gamma_{z}(\nu)$ must be symmetric, nonnegative, and with a finite integral over the interval $[-1 / 2,1 / 2]$. Conversely, any function satisfying these conditions can be considered to be the Fourier transform of the CF of a real signal.

Suppose now that $z[k]$ is complex. As $\Gamma_{z}(\nu)$ is the power spectrum, $\Gamma_{z}(\nu)$ must satisfy the same conditions as in the real case, except for the symmetry property. On the other hand, the stationarity implies that the RF is symmetric, and then, the same property appears for its Fourier transform, i.e., $R_{z}(\nu)=R_{z}(-\nu)$. However, notice that $R_{z}(\nu)$ is, in general, a complex function.

Let us introduce the $2 \times 1$ complex vector

$$
\boldsymbol{z}[k]=\left[z[k], z^{*}[k]\right]^{T} \text {. }
$$

Its $\mathrm{CF}$ is the $2 \times 2$ matrix $E\left(z[k] z^{H}[k-\tau]\right)$, where the symbol $H$ means transposition and complex conjugation. The Fourier transform of this matrix is the spectral matrix of $z[k]$ noted $\boldsymbol{\Gamma}_{\boldsymbol{z}}(\nu)$, and it is known (see [1, p. 235] and [2, p. 454]) that $\Gamma_{\boldsymbol{z}}(\nu)$ must be a nonnegative definite matrix. A simple calculation gives

$$
\Gamma_{\boldsymbol{z}}(\nu)=\left[\begin{array}{cc}
\Gamma_{z}(\nu) & R_{z}(\nu) \\
R_{z}^{*}(-\nu) & \Gamma_{z}(-\nu)
\end{array}\right]
$$

This matrix is nonnegative definite if and only if one of its diagonal elements and its determinant are nonnegative. This property is obvious for the diagonal elements. By using the symmetry of $R_{z}(\nu)$, the condition on the determinant yields

$$
\left|R_{z}(\nu)\right|^{2} \leq \Gamma_{z}(\nu) \Gamma_{z}(-\nu) .
$$

This is a necessary condition that the function $R_{z}(\nu)$ must satisfy in order to be the Fourier transform of an RF of a signal with power spectrum $\Gamma_{z}(\nu)$. We shall verify in Section IV that this condition is also sufficient.

It is now of interest to investigate the case when the upper bound of the inequality (5) is reached, which means that the rank of $\Gamma_{z}(\nu)$ is one. It is shown in Appendix A that the rank of $\Gamma z(\nu)$ is one for any value of $\nu$ if and only if the two components of the vector $z[k]$ can be deduced from each other by a linear filtering. This can be written as

$$
z^{*}[k]=\sum_{l} h[l] z[k-l] .
$$

We shall examine some examples of this situation in Section III.

\section{Nonstationary Signals}

Suppose now that the two moments (1) and (2) are not functions of $k_{1}-k_{2}$ only and that they have bounded Fourier transforms noted $\Gamma_{z}\left(\nu_{1}, \nu_{2}\right)$ and $R_{z}\left(\nu_{1}, \nu_{2}\right)$. Consider two linear filters $F_{A}$ and $F_{B}$ characterized by their frequency response $G_{A}(\nu)$ and $G_{B}(\nu)$, respectively. Let $z_{A}[k]$ be the output of $F_{A}$ when its input is $z[k]$, and let $Z_{A}$ be the random variable equal to $z_{A}[0]$. It results from the classical formulae of the harmonic analysis that

$$
E\left[\left|Z_{A}\right|^{2}\right]=\iint G_{A}\left(f_{1}\right) G_{A}^{*}\left(f_{2}\right) \Gamma_{z}\left(f_{1},-f_{2}\right) d f_{1} d f_{2} .
$$

The same relation can be obtained for $E\left[\left|Z_{B}\right|^{2}\right]$ by replacing $A$ with $B$. Furthermore, we have

$$
E\left[Z_{A} Z_{B}\right]=\iint G_{A}\left(f_{1}\right) G_{B}\left(f_{2}\right) R_{z}\left(f_{1}, f_{2}\right) d f_{1} d f_{2} .
$$

Suppose now that the frequency response $G_{A}(\nu)$ is equal to 1 for $\nu_{1}-\Delta \nu / 2<\nu<\nu_{1}+\Delta \nu / 2$ and 0 otherwise. In this case, (7) can be approximated for $\Delta \nu$ sufficiently small by

$$
E\left[\left|Z_{A}\right|^{2}\right]=\Gamma_{z}\left(\nu_{1},-\nu_{1}\right) \Delta \nu^{2}
$$

Introducing the same assumption for $G_{B}(\nu)$ where $\nu_{1}$ is replaced by $\nu_{2}$ yields

$$
E\left[Z_{A} Z_{B}\right]=R_{z}\left(\nu_{1}, \nu_{2}\right) \Delta \nu^{2}
$$

Applying the Schwarz inequality to $Z_{A}$ and $Z_{B}^{*}$ yields

$$
\left|R_{z}\left(\nu_{1}, \nu_{2}\right)\right|^{2} \leq \Gamma_{z}\left(\nu_{1},-\nu_{1}\right) \Gamma_{z}\left(\nu_{2},-\nu_{2}\right) .
$$

This is the transposition to the nonstationary case of (5).

\section{Wide-Sense Stationary Signals}

Assume now that $z[k]$ is WSS but not SOS. This means that there exists a power spectrum $\Gamma_{z}(\nu)$ and a function $R_{z}\left(\nu_{1}, \nu_{2}\right)$ that cannot be reduced to a function of only one frequency. This situation is much more complicated to analyze. First, note that $\Gamma_{z}\left(\nu_{1}, \nu_{2}\right)$ introduced above can be expressed as

$$
\Gamma_{z}\left(\nu_{1}, \nu_{2}\right)=\Gamma_{z}\left(\nu_{1}\right) \delta\left(\nu_{1}+\nu_{2}\right)
$$

where $\Gamma_{z}(\nu)$ is the power spectrum, and $\delta(\nu)$ is the Dirac distribution. This shows that $\left|\Gamma_{z}\left(\nu_{1}, \nu_{2}\right)\right|$ is no longer bounded 
and that $\Gamma_{z}\left(\nu_{1}, \nu_{2}\right)$ is zero in $[-1 / 2,1 / 2] \times[-1 / 2,1 / 2]$, except on the stationary manifold defined by $\nu_{1}+\nu_{2}=0$ (see $[1, \mathrm{p}$. 238], [11, ch. VI], or [12, p. 238]).

If $\left|R_{z}\left(\nu_{1}, \nu_{2}\right)\right|$ remains bounded, then (11) is always satisfied, and there is no specific condition on the function $R_{z}\left(\nu_{1}, \nu_{2}\right)$. Therefore, the only case of interest appears when $\left|R_{z}\left(\nu_{1}, \nu_{2}\right)\right|$ is not bounded. In order to avoid too sophisticated calculations, let us limit our study to the simplest situation that appears when

$$
R_{z}\left(\nu_{1}, \nu_{2}\right)=R_{z, c}\left(\nu_{1}, \nu_{2}\right)+R_{z}\left(\nu_{1}\right) \delta\left(\nu_{2}-h\left(\nu_{1}\right)\right)
$$

where $\left|R_{z, c}\left(\nu_{1}, \nu_{2}\right)\right|$ is bounded. This means that there is a continuous distribution in the plane $\nu_{1} \times \nu_{2}$ and another component distributed in the curve defined by $\nu_{2}=h\left(\nu_{1}\right)$. In the SOS case, we necessarily have $R_{z, c}\left(\nu_{1}, \nu_{2}\right)=0$ and $h(\nu)=-\nu$, which again introduces the stationary manifold $\nu_{1}+\nu_{2}=0$. Since the function $R_{z}\left(\nu_{1}, \nu_{2}\right)$ is symmetric, $R_{z, c}\left(\nu_{1}, \nu_{2}\right)$ must also be symmetric. For the same reason, the function $h(\nu)$ cannot be arbitrary. We deduce from (13) that the symmetry of $R_{z}\left(\nu_{1}, \nu_{2}\right)$ implies $h(\nu)=h^{-1}(\nu)$, which means that the curve $\nu_{2}=h\left(\nu_{1}\right)$ in the plane $\nu_{1} \times \nu_{2}$ is symmetric with respect to the straight line $\nu_{1}=\nu_{2}$ of the plane. Applying the same reasoning as in the previous subsection yields

$$
\left|R_{z}(\nu)\right|^{2} \leq \Gamma_{z}(\nu) \Gamma_{z}[h(\nu)] .
$$

This shows that there is no condition on the function $R_{z, c}\left(\nu_{1}, \nu_{2}\right)$, whereas the function $R_{z}(\nu)$ must satisfy (14). Notice that (14) is equivalent to (5) when $z[k]$ is SOS because, in this case, $h(\nu)=-\nu$.

\section{EXAMPLES}

In the previous section, several necessary conditions on the RF have been introduced, but no example of signals satisfying these conditions has been given. This is the purpose of this section. In particular, we present some examples of signals that are WSS and analytic without being circular.

\section{A. Degenerate Second-Order Stationary Signals}

Is it possible to find some signals satisfying (6)? They are called degenerate because the rank of (4) is equal to one instead of two. A trivial example is the case of real signals: They satisfy (6) with $h[k]=\delta[k]$. However, in a study devoted to complex signals, it is worth finding other examples.

For this, let us recall the classical expressions of linear filtering. If an SOS signal $w[k]$ is passed through a linear filter of frequency response $G(\nu)$, the Fourier transforms of the covariance and relation functions of the input $w[k]$ and the output $z[k]$ are related by

$$
\begin{aligned}
\Gamma_{z}(\nu) & =|G(\nu)|^{2} \Gamma_{w}(\nu), \\
R_{z}(\nu) & =G(\nu) G(-\nu) R_{w}(\nu) .
\end{aligned}
$$

In order to obtain (15b), we start from the convolution relation $z[k]=\Sigma_{l} h[l] w[k-l]$. As a result, the RF of $z[k]$ satisfies

$$
\begin{aligned}
r_{z}[p] & =E\{z[k] z[k-p]\} \\
& =\sum_{l_{1}, l_{2}} h\left[l_{1}\right] h\left[l_{2}\right] r_{w}\left[p+l_{2}-l_{1}\right] .
\end{aligned}
$$

Introducing the Fourier transform $R_{w}(\nu)$ of $r_{w}[k]$ yields

$$
\begin{aligned}
r_{z}[p] & =\int \sum_{l_{1}, l_{2}} h\left[l_{1}\right] h\left[l_{2}\right] R_{w}(\nu) e^{j 2 \pi \nu\left(p+l_{2}-l_{1}\right)} d \nu \\
& =\int G(\nu) G(-\nu) R_{w}(\nu) e^{j 2 \pi \nu p} d \nu
\end{aligned}
$$

which gives (15b).

Suppose now that $w[k]$ is real. This implies that $\Gamma_{w}(\nu)=$ $R_{w}(\nu)=\Gamma_{w}(-\nu)$. Suppose also that the filter is not real, which is characterized by $G(\nu) \neq G^{*}(-\nu)$. It is therefore clear that $z[k]$ cannot be real. However, we have $\left|R_{z}(\nu)\right|^{2}=$ $\Gamma_{z}(\nu) \Gamma_{z}(-\nu)$. Furthermore, we verify that (6) is satisfied with the filter of frequency response $H(\nu)=G^{*}(-\nu) / G(\nu)$. Therefore, by filtering a real signal in a nonreal filter, we obtain a complex signal satisfying (6).

\section{B. Analytic Signals}

As indicated above, the simplest example of a complex signal is the analytic signal. When it is WSS, it is characterized by the property $\Gamma_{z}(\nu)=0$ for $\nu<0$. This property does not imply that it is SOS.

Assume that the analytic signal $z[k]$ is SOS. In this case, its second-order properties are characterized by $\Gamma_{z}(\nu)$ and $R_{z}(\nu)$ introduced in the previous section. We can then apply (5), and since $\Gamma_{z}(\nu)=0$ for $\nu<0$, we deduce that

$$
R_{z}(\nu)=0 .
$$

This means that the RF defined by (2) is zero. Thus, we deduce that if a random analytic signal is SOS, it is second-order circular.

More generally, this property of zero RF, or of secondorder circularity, holds for any SOS signal $z[k]$ satisfying $\Gamma_{z}(\nu) \Gamma_{z}(-\nu)=0$, and the analytic signal is only a particular case of this relation. Notice that $\Gamma_{z}(\nu) \Gamma_{z}(-\nu)=0$ implies that the $\mathrm{CF}$ cannot be real. Indeed, if this were true, we would have $\Gamma_{z}(\nu)=\Gamma_{z}(-\nu)$, which is impossible when $\Gamma_{z}(\nu) \Gamma_{z}(-\nu)=0$.

Suppose now that the analytic signal $z[k]$ is WSS but not SOS. In this case, the signal is not necessarily circular. Let us present a simple example of this situation. Let $x[k]$ be a real WSS signal with a bandlimited power spectrum. This means that the power spectrum $\Gamma_{x}(\nu)$ is zero for $|\nu| \geq B$. Consider the signal $z[k]=\exp \left(j 2 \pi \nu_{0} k\right) x[k]$ with $\nu_{0}>B$. This condition implies that $z[k]$ is an analytic signal. However, its RF is

$$
r_{z}\left[k_{1}, k_{2}\right]=e^{j 2 \pi \nu_{0}\left(k_{1}+k_{2}\right)} \gamma_{x}\left[k_{1}-k_{2}\right]
$$

where $\gamma_{x}[p]$ is the CF of $x[k]$. By Fourier transformation, we immediately obtain

$$
R_{z}\left(\nu_{1}, \nu_{2}\right)=\Gamma_{x}\left(\nu_{1}-\nu_{0}\right) \delta\left(\nu_{1}+\nu_{2}-2 \nu_{0}\right)
$$


which has the structure (13) with $R_{z, c}\left(\nu_{1}, \nu_{2}\right)=0$, and $h(\nu)=2 \nu_{0}-\nu$. This function satisfies the symmetry property $h(\nu)=h^{-1}(\nu)$. Noting that $\Gamma_{z}(\nu)=\Gamma_{x}\left(\nu-\nu_{0}\right)$, the condition (14) becomes

$$
\Gamma_{x}^{2}\left(\nu-\nu_{0}\right) \leq \Gamma_{x}\left(\nu-\nu_{0}\right) \Gamma_{x}\left(\nu_{0}-\nu\right) .
$$

As $\Gamma_{x}(\nu)$ is a symmetric function, (21) is satisfied and becomes an equality. This does not mean that (6) is satisfied because this equation was shown in the SOS case. However, as $x[k]$ is real, we have

$$
z^{*}[k]=e^{-j 4 \pi \nu_{0} k} z[k]
$$

which introduces a linear time dependent relation between $z[k]$ and $z^{*}[k]$. We then have an example of a WSS analytic signal that is not circular. Note at this stage that the real and imaginary parts of $z[k]$ are no longer stationary. Indeed, the real part is $\cos \left(2 \pi \nu_{0} k\right) x[k]$ and is not stationary, even if $z[k]$ has this property.

\section{Signals Satisfying (13)}

Let us now present an example of a non SOS signal, the $\mathrm{RF}$ of which has the structure (13). In order to introduce some diversity in the presentation, we describe this signal in the CT case. There exists, of course, an obvious DT version of it. Let $x(t)$ and $y(t)$ be two zero-mean SOS complex signals, and consider the signal

$$
z(t)=A e^{j 2 \pi F t} x(t)+y(t) .
$$

In this equation, $A$ is a real random amplitude, and we assume that $E(A)=0$ and $E\left(A^{2}\right)=1$. Similarly, $F$ is a random frequency. We denote $p(f)$ and $\phi(u)$ to be the probability density function and the characteristic function of $F$, respectively. Furthermore, we assume that $A, F, x(t)$, and $y(t)$ are independent. It is clear that $E[z(t)]=0$. From all these assumptions, we find that $z(t)$ is WSS, and its CF is

$$
\gamma_{z}(\tau)=E\left[z(t) z^{*}(t-\tau)\right]=\phi(2 \pi \tau) \gamma_{x}(\tau)+\gamma_{y}(\tau) .
$$

It results from (24) that the power spectrum takes the form

$$
\Gamma_{z}(\nu)=\int p(f) \Gamma_{x}(\nu-f) d f+\Gamma_{y}(\nu) .
$$

On the other hand, the RF is

$$
r_{z}\left(t_{1}, t_{2}\right)=\phi\left[2 \pi\left(t_{1}+t_{2}\right)\right] r_{x}\left(t_{1}-t_{2}\right)+r_{y}\left(t_{1}-t_{2}\right)
$$

and by Fourier transformation, we obtain

$$
\begin{aligned}
R_{z}\left(\nu_{1}, \nu_{2}\right)= & \frac{1}{2} p\left(\frac{\nu_{1}+\nu_{2}}{2}\right) R_{x}\left(\frac{\nu_{1}-\nu_{2}}{2}\right) \\
& +R_{y}\left(\nu_{1}\right) \delta\left(\nu_{1}+\nu_{2}\right) .
\end{aligned}
$$

This expression has the structure (13) with $R_{z}(\nu)=R_{y}(\nu)$ and $h(\nu)=-\nu$. Since $y(t)$ is SOS, the pair $\left[R_{y}(\nu), \Gamma_{y}(\nu)\right]$ satisfies (5). Then, it results from (25) that

$$
\begin{aligned}
\left|R_{z}(\nu)\right|^{2} & =\left|R_{y}(\nu)\right|^{2} \leq \Gamma_{y}(\nu) \Gamma_{y}(-\nu) \leq \Gamma_{z}(\nu) \Gamma_{z}(-\nu) \\
& \leq \Gamma_{z}(\nu) \Gamma_{z}[h(\nu)] .
\end{aligned}
$$

Hence, we check that (14) is satisfied.

\section{Modeling Problem}

The inequality (5) introduced in Section II is a necessary condition for the pair of functions $\left[\Gamma_{z}(\nu), R_{z}(\nu)\right]$ to be the Fourier transforms of the $\mathrm{CF}$ and the RF of an SOS signal $z[k]$. The purpose of this section is to show that this condition is also sufficient. This means that to a given pair of functions $[\Gamma(\nu), R(\nu)]$ satisfying the three following conditions:

$$
\begin{aligned}
\Gamma(\nu) & \geq 0 \\
R(\nu) & =R(-\nu) \\
|R(\nu)|^{2} & \leq \Gamma(\nu) \Gamma(-\nu)
\end{aligned}
$$

it is possible to associate at least one random signal such that the Fourier transforms of its $\mathrm{CF}$ and $\mathrm{RF}$ are $\Gamma(\nu)$ and $R(\nu)$, respectively. Finding such a signal is realized by a modeling approach.

This approach is well known in the real case. It consists of showing that to any symmetric and nonnegative function $\Gamma(\nu)$, it is possible to associate at least one signal such that $\Gamma(\nu)$ is its power spectrum. The solution is obtained by a linear procedure. Indeed, passing a unit white noise through a linear filter with frequency response $\sqrt{\Gamma(\nu)}$ gives an output with a power spectrum equal to $\Gamma(\nu)$.

Extending this method to the complex case requires that the concept of complex white noise be introduced first.

\section{A. Second-Order White Noises}

A WSS signal $z[k]$ is said to be white if its power spectrum is constant. In terms of its $\mathrm{CF}$, this condition is equivalent to

$$
\gamma_{z}[k, k-m]=\gamma_{z}[m]=\gamma_{z} \delta[m]
$$

where $\gamma_{z}$ is the variance of the signal, and $\delta[\cdot]$ the Kroneckerdelta function. However, whiteness does not introduce any property on the RF that can even be nonstationary. This implies that there are different kind of white noises. Now, if SO stationarity is introduced, the RF must satisfy the constraint (5), which takes the form

$$
\left|R_{z}(\nu)\right|^{2} \leq \gamma_{z}^{2} .
$$

In order to avoid this freedom concerning $R_{z}(\nu)$, it is often assumed that whiteness implies that $R_{z}(\nu)$ is equal to zero [2]. In this case, which remains particular, it is better to use the expression of circular white noise. It is thus easy to deduce from (30) and from the fact that the RF is zero that the real and imaginary parts of $z[k]$ are uncorrelated and white. The introduction of circularity especially appears in the studies using the concept of complex Gaussian white noise. In [2, p. 54], it is explicitly indicated in the definition of a complex white Gaussian noise that its RF is zero or that a complex Gaussian white noise is circular, which is equivalent. More generally, the circularity is introduced in the definition of a complex Gaussian stochastic process in many excellent books such as [7, p. 72] and [13], which is entirely devoted to this question, and [14, vol. 2, p. 128], where the term strongly normal is used instead of circular normal.

Another conception of whiteness consists of saying that it involves only instantaneous relationships. This means that the 
RF must have a structure similar to (30) or that

$$
r_{z}[m]=r_{z} \delta[m]
$$

where $r_{z}$ can be complex but must satisfy $\left|r_{z}\right| \leq \gamma_{z}$ as a consequence of (5). Because of the analogy between (30) and (32), this kind of whiteness will be called double whiteness in what follows.

Note that, contrary to the $\mathrm{CF}$, the RF can be equal to zero for $m=0$. The simplest example is the case of a white noise for which $r_{z}[m]$ is nonzero for one value of $m$ only. Because of the symmetry, $r_{z}[m]$ can be written as

$$
r_{z}[m]=\mu_{z}\{\delta[m-d]+\delta[m+d]\}
$$

where $\mu_{z}$ is a complex number, and $d$ is a positive integer. In this case, $R_{z}(\nu)$ takes the form

$$
R_{z}(\nu)=2 \mu_{z} \cos (2 \pi \nu d)
$$

and (31) becomes

$$
\left|\mu_{z}\right|^{2} \leq(1 / 4) \gamma_{z}^{2}
$$

Let us verify that it is possible to construct a white signal with this RF. Suppose that $w[k]$ is a circular unit white noise. This means that $\gamma_{w}=1$. Let $z[k]$ be the signal

$$
z[k]=\left(1+|a|^{2}\right)^{-1 / 2}\left\{w[k]+a w^{*}[k-d]\right\}
$$

where $a$ is a complex number. It is obvious that $z[k]$ remains a unit white noise. However, its RF is given by (33), where

$$
\mu_{z}=a\left(1+|a|^{2}\right)^{-1} \text {. }
$$

It is clear that $\left|\mu_{z}\right| \leq 1 / 2$, and the upper bound is obtained when $|a|=1$.

\section{B. Linear Modeling}

As one of the advantages of linear models is, above all, their simplicity, it seems appropriate to extend the linear procedure used in the real case to the complex one. We shall now prove that this is not possible.

At first, the same argument of simplicity results in the assumption that the driving noise $w[k]$ is doubly white or characterized by the parameters $\gamma_{w}$ and $r_{w}$ appearing in (30) and (32), where $z[k]$ is replaced by $w[k]$. This means that $\Gamma_{w}(\nu)=\gamma_{w}$ and $R_{w}(\nu)=r_{w}$, and without any loss of generality, we can assume that $\gamma_{w}=1$, which implies that $\left|r_{w}\right| \leq 1$.

Consider now an arbitrary pair of functions $[\Gamma(\nu), R(\nu)]$ satisfying (29). We are looking for a complex linear filter with frequency response $G(\nu)$ and a doubly white noise characterized by $r_{w}$ such that the Fourier transforms of the $\mathrm{CF}$ and the RF of the output are $\Gamma(\nu)$ and $R(\nu)$, respectively. Applying (15) yields

$$
\begin{aligned}
|G(\nu)|^{2} & =\Gamma(\nu), \\
r_{w} G(\nu) G(-\nu) & =R(\nu) .
\end{aligned}
$$

These relations imply

$$
|R(\nu)|^{2}=\left|r_{w}\right|^{2} \Gamma(\nu) \Gamma(-\nu) .
$$

Consequently, if the pair $[\Gamma(\nu), R(\nu)]$ satisfies (29) but not (39), the linear procedure fails. A simple example of this situation appears when $\Gamma(\nu)=1$ and $R(\nu)$ is not constant but such that $|R(\nu)|^{2} \leq 1$. This is the case with the white noise $z[k]$ defined by (36) for example.

On the other hand, there are cases where the linear procedure can be applied. This appears, for instance, when (29c) becomes an equality, which corresponds to the degenerate case studied in Section III-A. Another specific situation appears when $R(\nu)=0$. In this case, it suffices to take $r_{w}=0,|G(\nu)|^{2}=$ $\Gamma(\nu)$, and the modeling is then made possible by using a circular white noise. Hence, when $R(\nu)=0$, the modeling problem can be solved exactly as in the real case. This justifies the importance of the assumption of circularity.

In conclusion, by using a linear procedure, it is not possible to extend the method used for real signals to prove that the conditions (29) are sufficient in the sense indicated at the beginning of this section. In the next subsection, we show that this becomes possible by using another class of linear systems.

\section{Widely Linear Modeling}

Recently, it was shown in [15] and [16] that for processing complex signals, it is of interest to use widely linear systems. The input-output relationship of such systems is

$$
z[k]=\sum g[l] w[k-l]+\sum h[l] w^{*}[k-l] .
$$

This means that $z[k]$ is the sum of the two signals, respectively, obtained by a linear filtering of $w[k]$ and of $w^{*}[k]$. It is clear that (40) is not a linear filtering in the classical sense. Indeed, if $z_{1}[k]$ and $z_{2}[k]$ are the outputs associated with the inputs $w_{1}[k]$ and $w_{2}[k]$, respectively, the output associated with $w_{1}[k]+w_{2}[k]$ is $z_{1}[k]+z_{2}[k]$. This introduces a kind of linearity. However, the output associated with $\lambda w[k]$ is generally not $\lambda z[k]$ when $\lambda$ is a complex number, which is a necessary property of classical linear filters. On the other hand, the second-order characteristics of the output $z[k]$ of (40) can be obtained from those of the input $w[k]$. These properties justify the denomination of widely linear systems. As (40) introduces a large degree of freedom because of the introduction of two linear filters, we shall restrict the degree of freedom on the input and assume that $w[k]$ is circular and white with a unit power $\left(\gamma_{w}=1\right)$. As said above, this assumption is almost always introduced when talking about complex white noises. Let us call $G(\nu)$ and $H(\nu)$ the Fourier transform of $g[k]$ and $h[k]$, respectively. The Fourier transforms of the CF and the RF of $z[k]$ given by (40) are calculated in Appendix B. In the modeling procedure, these functions must be equal to $\Gamma(\nu)$ and $R(\nu)$, respectively, which gives

$$
\begin{aligned}
|G(\nu)|^{2}+|H(\nu)|^{2} & =\Gamma(\nu) \\
G(\nu) H(-\nu)+G(-\nu) H(\nu) & =R(\nu) .
\end{aligned}
$$

It is worth pointing out that (36) is a particular case of (40) with $G(\nu)=\left(1+|a|^{2}\right)^{-1 / 2}$ and $H(\nu)=a(1+$ $\left.|a|^{2}\right)^{-1 / 2} \exp (-j 2 \pi \nu d)$. It is then easy to check that (41) and (42) are verified when $\Gamma(\nu)=1$, and $R(\nu)$ is given by (34) and (37). 
Coming back to the modeling problem, we now have to show that if $[\Gamma(\nu), R(\nu)]$ satisfy (29), there is at least one pair $[G(\nu), H(\nu)]$ that is the solution to (41) and (42). Note that (41) and (42) obviously imply that (29a) and (29b) are satisfied. Now, consider an arbitrary frequency $\nu$ in $[-1 / 2,1 / 2]$, and let us assume that

$$
G(\nu) H(-\nu)=(1 / 2) R(\nu)=G(-\nu) H(\nu) .
$$

Then, by multiplying (41) by $|G(-\nu)|^{2}$, we obtain

$$
|G(\nu)|^{2}|G(-\nu)|^{2}+(1 / 4)|R(\nu)|^{2}=\Gamma(\nu)|G(-\nu)|^{2}
$$

and by using the symmetry of $R(\nu)$, we deduce that

$$
\Gamma(\nu)|G(-\nu)|^{2}=\Gamma(-\nu)|G(\nu)|^{2} .
$$

By multiplying (44) by $\Gamma(\nu)$, we get

$$
\begin{gathered}
\Gamma(-\nu)|G(\nu)|^{4}-\Gamma(\nu) \Gamma(-\nu)|G(\nu)|^{2} \\
+(1 / 4) \Gamma(\nu)|R(\nu)|^{2}=0 .
\end{gathered}
$$

If $\Gamma(-\nu)=0$, then, according to $(29 \mathrm{c}), R(\nu)=0$. In this case, we take $G(-\nu)=H(-\nu)=0$. Then, (42) is satisfied, and the problem consists of solving (41), which can be done easily. Take, for instance, $H(\nu)=0$, and define $G(\nu)$ by $|G(\nu)|^{2}=\Gamma(\nu)$. This equation is not sufficient to determine $G(\nu)$ because the phase is unknown. However, for the modeling problem, we only have to show that there exists at least one solution. In the same way, if $\Gamma(\nu)=0, R(\nu)=0$. Then, we take $G(\nu)=H(\nu)=0$ so that (42) is satisfied, and we can choose $H(-\nu)=0$ and define $G(-\nu)$ by $|G(-\nu)|^{2}=\Gamma(-\nu)$.

Assume now that $\Gamma(\nu) \Gamma(-\nu) \neq 0$. Then, (46) is a secondorder polynomial equation in $|G(\nu)|^{2}$. The sum and the product of the roots being positive, (46) admits two positive solutions if and only if

$$
D(\nu)=\Gamma(\nu) \Gamma(-\nu)\left[\Gamma(\nu) \Gamma(-\nu)-|R(\nu)|^{2}\right] \geq 0 .
$$

Due to (29a) and (29c), this inequality is satisfied.

When $D(\nu)>0$, there are two distinct positive roots for (46): $\left|G_{1}(\nu)\right|^{2}$ and $\left|G_{2}(\nu)\right|^{2}$. Any one of these roots can be taken as a value for $|G(\nu)|^{2}$, whereas the phase of $G(\nu)$ is arbitrary. Next, $H(\nu)$ is completely determined by (43). Take, for instance, $|G(\nu)|=\left|G_{1}(\nu)\right|$. It results from (43) that $H(\nu)=H_{1}(\nu)$, where $H_{1}(\nu)$ satisfies

$$
\left|G_{1}(-\nu)\right|^{2}\left|H_{1}(\nu)\right|^{2}=(1 / 4)|R(\nu)|^{2} .
$$

Now, as a consequence of (46),

$$
\left|G_{1}(\nu)\right|^{2}\left|G_{2}(\nu)\right|^{2}=(1 / 4) \Gamma(\nu)|R(\nu)|^{2} / \Gamma(-\nu) .
$$

Hence, we deduce from (48), (49), and (45) that $\left|H_{1}(\nu)\right|=$ $\left|G_{2}(\nu)\right|$. Therefore, $|H(\nu)|=\left|G_{2}(\nu)\right|$, whereas the phase of $H(\nu)$ is determined by (43).

Let us now consider the case where $D(\nu)=0$, which means that $(29 \mathrm{c})$ becomes an equality. In this case, we obtain from (46) that $|G(\nu)|^{2}=(1 / 2) \Gamma(\nu)$, and $H(\nu)$ is still defined by (43). It is easy to verify that $|H(\nu)|=|G(\nu)|$.

In conclusion, any pair of functions $\Gamma(\nu)$ and $R(\nu)$ satisfying the three conditions (29) can be considered to be the
Fourier transform of the $\mathrm{CF}$ and $\mathrm{RF}$ of the output of at least one widely linear filter driven by a circular unit white noise. This solves the modeling problem and shows that the three previous conditions are necessary and sufficient for the second-order statistics of an SOS complex signal.

Finally, note that the modeling of any complex signal from a circular unit white noise is not unique, and, as in the case of real signals, it is possible to reduce the degree of freedom by introducing some constraints such as the causality for example. We shall analyze this point by studying prediction problems and generalizing the concept of complex autoregressive (AR) signals.

\section{Autoregressive Modeling AND PREdiction}

Complex AR models are widely used in signal processing and especially in spectral analysis (see [2, chs. 5 and 6]). However, it is almost always assumed that the driving white noise is circular, which leads to a zero relation function. The purpose of this section is to examine the second-order properties of AR signals and, especially, to calculate the RF and apply the results to prediction problems.

An AR signal $z[k]$ is defined by the difference equation

$$
z[k]=\boldsymbol{a}^{H} \boldsymbol{Z}[k]+w[k]
$$

where $\boldsymbol{a}$ is the regression vector with components $a_{i}, 1 \leq i \leq$ $p, Z[k]$ is the past vector with components $z[k-i], 1 \leq i \leq p$, and $w[k]$ is a driving noise. We assume that $w[k]$ is SOS and white in the sense that its power spectrum is constant, $\Gamma_{w}(\nu)=\gamma_{w}$. Nevertheless, we do not make any assumption on its RF $r_{w}[k]$. All the terms appearing in (50) are usually complex, and the vector $\boldsymbol{a}$ is such that the filter defined by (50) is stable. As a consequence, the signal $z[k]$ is SOS, and its second-order properties are entirely specified by those of $w[k]$ and by the regression vector.

\section{A. Autoregressive Modeling}

The purpose of AR modeling is to find an AR model of a given order $p$ such that its second-order properties are a good approximation of those of an observed signal. Suppose that the $\mathrm{CF} \gamma[k]$ of the observed signal is known for $0 \leq k \leq p$ and that its RF $r[k]$ is known for any $k$. The problem is then to find the vector $\boldsymbol{a}$ appearing in (50), the variance $\gamma_{w}$ of $w[k]$, and the relation function $r_{w}[k]$ of $w[k]$ such that $\gamma_{z}[k]=\gamma[k]$ and $r_{z}[k]=r[k]$, where $z[k]$ is the output of (50).

Let $\boldsymbol{\Gamma}$ be the covariance matrix $E\left(Z[k] Z^{H}[k]\right)$, and let $\boldsymbol{c}$ be the correlation vector $E\left(z^{*}[k] Z[k]\right)$. The elements of $\boldsymbol{\Gamma}$ and $c$ are defined by the values $\gamma_{z}[i]$ for $0 \leq i \leq p$ and are identified to the corresponding values of $\gamma[i]$. We deduce from (50) that

$$
\begin{aligned}
\boldsymbol{\Gamma} \boldsymbol{a} & =\boldsymbol{c}, \\
\gamma_{w} & =\gamma[0]-\boldsymbol{a}^{H} \boldsymbol{c} .
\end{aligned}
$$

Then, the determination of $\boldsymbol{a}$ and $\gamma_{w}$ can be obtained by the standard normal equations (51). It is worth pointing out that these equations are independent of the structure of the RF $r_{w}[k]$.

Without any a priori knowledge or assumption on $r_{w}[k]$, this function can be determined by using (15b). Indeed, as the 
vector $\boldsymbol{a}$ is determined from (51), the linear filter defined by (50) is also determined, and its transfer function $G(z)$ is equal to $[P(z)]^{-1}$ with

$$
P(z)=1-\sum_{k=1}^{p} a_{k}^{*} z^{-k} .
$$

Written in the $z$ domain, (15b) can be expressed as

$$
R_{w}(z)=P(z) P\left(z^{-1}\right) R_{z}(z)=F(z) R_{z}(z) .
$$

It results from (52) that

$$
F(z)=\sum_{k=-p}^{p} c_{k} z^{-k}
$$

where the coefficients $c_{k}$ can easily be deduced from the $a_{k} \mathrm{~s}$. As $R_{z}(z)$ has the structure (54), where the sum is, in general, an infinite series, and $c_{k}$ is replaced by $r_{z}[k]$, we deduce that the RF $r_{w}[k]$ can easily be deduced from the coefficients $c_{k}$ and the observed RF $r[k]$ using (53).

In reality, this very general method can be greatly simplified if some assumptions on $r_{w}[k]$ can be introduced. The simplest consists of saying that the driving noise $w[k]$ is doubly white or that (30) and (32), where $z[k]$ is replaced by $w[k]$, are valid. However, this assumption cannot be arbitrarily introduced, and its validity must be verified. Indeed, when (32) is valid, we deduce from (50) that

$$
\begin{aligned}
\boldsymbol{R} \boldsymbol{a}^{*} & =\boldsymbol{r}, \\
r_{w} & =r[0]-\boldsymbol{a}^{H} \boldsymbol{r}
\end{aligned}
$$

where $\boldsymbol{R}=E\left(\boldsymbol{Z}[k] Z^{T}[k]\right)$ and $\boldsymbol{r}=E\left(z[k] \boldsymbol{Z}^{T}[k]\right)$. These quantities are entirely determined from the values of the observed RF $r[i]$ for $0 \leq i \leq p$. Therefore, it is clear that (55b) can give the value of $r_{w}$ appearing in (32), provided that the vector $\boldsymbol{a}$ satisfies simultaneously (51) and (55). This gives a new illustration of the fact discussed in the previous section that a given signal generally cannot be modeled as the output of a linear filter driven by a doubly white noise.

\section{B. Prediction}

AR modeling is strongly related to prediction problems. If, in (50), $\boldsymbol{a}$ and $w[k]$ are real, $z[k]$ is also real, and its best mean square linear prediction in terms of its entire past is $\boldsymbol{a}^{T} \boldsymbol{Z}[k]$. Suppose now that, as previously, $\boldsymbol{a}$ is complex, and $w[k]$ is a complex white noise characterized by its variance $\gamma_{w}$ and by its RF $r_{w}[k]$. By using the finite past of order $p$, i.e. the vector $Z[k]$, it is possible to introduce two linear predictors. The first is the strictly linear predictor defined by $\hat{z}_{L}[k]=\boldsymbol{a}^{H} \boldsymbol{Z}[k]$. The second is the widely linear predictor (see [15]) defined by

$$
\hat{z}_{W L}[k]=\boldsymbol{u}^{H} \boldsymbol{Z}[k]+\boldsymbol{v}^{H} \boldsymbol{Z}^{*}[k] .
$$

Of course, the mean square error obtained with the widely linear predictor is always less than or equal to the one obtained with the strictly linear predictor. Our purpose in the following is twofold. First, we show that the two predictors are equal if the driving noise $w[k]$ is doubly white. Second, when $w[k]$ is not doubly white, we quantify the difference between the two mean square errors in the simple case of an AR(1) model.
Assume that $w[k]$ is doubly white. Let $\tilde{z}_{L}[k]=z[k]-$ $\hat{z}_{L}[k]$ be the strictly linear innovation. Since $\hat{z}_{L}[k]=$ $\boldsymbol{a}^{H} \boldsymbol{Z}[k], \tilde{z}_{L}[k]=w[k]$. Therefore, it results from (50) that $\tilde{z}_{L}[k]$ is uncorrelated with $z[k-i]$ and $z^{*}[k-i]$ for any positive integer $i$. Hence, $\tilde{z}_{L}[k]$ is orthogonal to $Z[k]$ and $Z^{*}[k]$. Then, $\tilde{z}_{L}[k]=\tilde{z}_{W L}[k]$, which is equivalent to $\hat{z}_{L}[k]=\hat{z}_{W L}[k]$. Therefore, the advantage of the widely linear procedure disappears when $w[k]$ is doubly white. It is worth presenting some comments on this result. It is known that when the assumption of circularity is valid, widely linear mean square estimation does not introduce any advantage with respect to strictly linear estimation [15]. The fact that circularity is very often introduced explicitly or implicitly justifies that the procedure of linear mean square estimation used for real signals can be transposed to the complex case. However, it was shown in [15] that the assumption of circularity is only a sufficient condition for the equivalence between linear and widely linear procedure. The case of prediction of complex AR signals shows that this equivalence is also valid for noncircular signals, provided that the driving noise is doubly white. Indeed, when $w[k]$ is doubly white but not circular $\left(R_{w}(z)=r_{w} \neq 0\right)$, it results from (53) that $z[k]$ is not circular $\left(R_{z}(z) \neq 0\right)$. As a conclusion, the only possibility to find a difference between the two procedures appears when the driving white noise is no longer doubly white.

Assume now that $w[k]$ is not doubly white. In order to quantify the difference between strict and widely linear prediction without using calculations that are too complicated, we shall now restrict our analysis to AR(1) signals. This means that the regression term in (50) is now $a^{*} z[k-1]$, where $|a|<1$. Then, $\hat{z}_{L}[k]=a^{*} z[k-1]$, and the strictly linear prediction error is the variance $\gamma_{w}$ of the driving noise $w[k]$. The widely linear predictor is given by (56), where $Z[k]$ is replaced by $z[k-1]$, and the predictor is now defined by two complex numbers $u$ and $v$. These numbers are calculated by writing that $\tilde{z}_{W L}[k]$ is orthogonal to $z[k-1]$ and its conjugate. This leads to the system of equations

$$
\begin{aligned}
& \gamma_{0} u+r_{0} v=\gamma_{1}^{*} \\
& r_{0}^{*} u+\gamma_{0} v=r_{1}^{*}
\end{aligned}
$$

where, for notational convenience, we noted that $\gamma_{i}=\gamma_{z}[i]$, and $r_{i}=r_{z}[i]$. Let $\Delta$ be the determinant $\gamma_{0}^{2}-\left|r_{0}\right|^{2}$. As a consequence of the Schwarz inequality applied to the pair $\left(z[k], z^{*}[k]\right), \Delta \geq 0$, and $\Delta=0$ if and only if $z[k]$ and $z^{*}[k]$ are linearly dependent.

Assume that $\Delta=0$. Because of the stationarity, the linear dependence between $z[k]$ and $z^{*}[k]$ can be written $z^{*}[k]=$ $\exp (j \phi) z[k]$, where $\phi$ is a real nonrandom number. Then, $\hat{z}_{W L}[k]=u^{*} z[k-1]+v^{*} z^{*}[k-1]=\left(u^{*}+v^{*} \exp (j \phi)\right) z[k-1]$, and we deduce immediately that $\hat{z}_{W L}[k]=\hat{z}_{L}[k]=a^{*} z[k-1]$. This provides another example of a situation where there is no interest to introduce (56) in terms of the mean square error.

Assume now that $\Delta \neq 0$. Then, $u$ and $v$ can be determined from (57) and (58). In particular, $v$ is given by

$$
v=\Delta^{-1}\left(\gamma_{0} r_{1}^{*}-r_{0}^{*} \gamma_{1}^{*}\right)
$$


The corresponding mean square error is given by $\epsilon_{W L}^{2}=$ $E\left(|z[k]|^{2}\right)-E\left(|\hat{z}[k]|^{2}\right)$, and a simple calculation yields

$$
\epsilon_{W L}^{2}=\gamma_{0}-\left(u \gamma_{1}+v r_{1}\right)
$$

From a calculation presented in Appendix C, we obtain

$$
\delta \epsilon^{2} \triangleq \epsilon_{L}^{2}-\epsilon_{W L}^{2}=\left(1 / \gamma_{0}\right) \Delta|v|^{2} .
$$

As $\gamma_{0}$ and $\Delta$ are positive, this difference is zero only when $v=0$. In this case, $\hat{z}_{W L}[k]$ is strictly linear. Otherwise, $\hat{z}_{W L}[k]$ always yields better results than $\hat{z}_{L}[k]$.

Note that $u$ and $v$ have been calculated in terms of the $\mathrm{CF}$ and the RF of the signal $z[k]$. However, as in the real case, it is necessary to express the widely linear predictor and its performance in terms of the parameters defining the model. An AR(1) signal defined by (50) is characterized at the second order by the complex regression parameter $a$ and by the variance $\gamma_{w}$ and the RF $r_{w}[k]$ of the driving noise $w[k]$. In the following, we denote $r_{w}[k]=\gamma_{w} \rho_{k}$ for any $k$. It results from the Schwarz inequality applied with the pair $\left(w[0], w^{*}[k]\right)$ that $\left|\rho_{k}\right| \leq 1$ for all $k$. It is shown in Appendix D that

$$
\begin{aligned}
& \gamma_{0}=\gamma_{w}\left[1-|a|^{2}\right]^{-1} \\
& \gamma_{1}=a^{*} \gamma_{0} .
\end{aligned}
$$

These relations correspond to the normal equations (51). The results are more complicated for the RF of $z[k]$ because $w[k]$ is not assumed to be doubly white. Let $T$ be the quantity

$$
T=\sum_{l=0}^{\infty}\left(a^{*}\right)^{l} \rho_{l+1} .
$$

It is shown in Appendix D that

$$
\begin{aligned}
& r_{0}=\gamma_{w}\left(1-a^{* 2}\right)^{-1}\left(\rho_{0}+2 a^{*} T\right) \\
& r_{1}=a^{*} r_{0}+\gamma_{w} T .
\end{aligned}
$$

Then, $u$ and $v$ can be explicitly calculated in terms of the parameters of the model using (62), (63), (65), and (66).

Let us now give some examples of applications of these expressions. Suppose first that $w[k]$ is doubly white. This is characterized by the fact that $\rho_{k}=0$ if $k \neq 0$. As a consequence $T=0$, and we easily verify from (59) that $v=0$. Therefore, in this particular case, we verify the general result, which has been indicated previously, that $\hat{z}_{W L}[k]$ is strictly linear. Assume now that $w[k]$ is not doubly white. In this case, the main difficulty is the calculation of $T$ defined by (64). However, this becomes very easy if the RF of $w[k]$ is given by (33), where $z[k]$ is replaced by $w[k]$. Indeed, in this case, the series (64) contains only one term. Suppose then that $d=1$ in (33), and to simplify further, assume that the coefficient $a$ of the AR(1) model is real. Then, the parameters of the model are $\gamma_{w}, a$, and $\mu_{w}$, which appears in (33). By using all the previous relations and after simple algebra, we obtain

$$
\delta \epsilon^{2}=\gamma_{w}\left(1-a^{2}\right) \frac{x}{1-4 a^{2} x}
$$

where $x=\left|\mu_{w}\right|^{2} / \gamma_{w}^{2}$. Because of (35), the parameter $x$ satisfies $0 \leq x \leq 1 / 4$. If $x=0$, the white noise is circular, and the interest of the widely linear prediction disappears. On the other hand, if $x=1 / 4$, which is its maximum possible value, $\delta \epsilon^{2}=\gamma_{w} / 4$, which is the maximum value of the gain of the widely linear prediction with respect to the strictly linear one. As $\epsilon_{L}^{2}=\gamma_{w}$, we deduce that $\epsilon_{W L}^{2}=(3 / 4) \gamma_{w}$. This very simple example of an $\operatorname{AR}(1)$ signal shows the interest of taking the RF into account in prediction problems. It illustrates the fact that the CF is not sufficient to calculate the optimal prediction. The same result can be obtained with more complicated models.

\section{CONCLUSION}

In order to describe the second-order properties of complex random signals completely, it is necessary to use two moments. The first is the classical covariance function, and the second is the relation function defined by (2). There are only very few results known on this function and on its use in signal processing problems. Our first result is to show that this function is not arbitrary. As soon as the covariance function is given, the Fourier transform of the relation function must satisfy some conditions that are given both for stationary and nonstationary signals. In order to show that these conditions are sufficient, we have solved the modeling problem of secondorder stationary signals. It consists of showing that any such signal can be considered to be the output of a widely linear system driven by a circular white noise. Incidentally, this approach shows that, in general, a stationary complex secondorder signal cannot be modeled as the output of a linear system driven by a white noise, as in the real case. Finally, these results are applied in prediction problems with autoregressive signals. We have shown that if the driving noise is circular or doubly white, the results known for real signals can be applied without making any difference. On the other hand, if these conditions are not fulfilled, a prediction procedure using a widely linear predictor can yield better results than the classical linear procedure. The advantage of the first method is analyzed in the very simple case of prediction of an AR(1) signal.

\section{APPENDIX A}

Let $x[k]$ and $y[k]$ be two zero-mean jointly SOS complex random signals. The spectral matrix of the random vector $[x[k], y[k]]^{T}$ is

$$
\Gamma(\nu)=\left[\begin{array}{cc}
\Gamma_{x}(\nu) & \Gamma_{x y}(\nu) \\
\Gamma_{y x}(\nu) & \Gamma_{y}(\nu)
\end{array}\right]
$$

where $\Gamma_{x}(\nu)$ and $\Gamma_{y}(\nu)$ are the power spectra of $x[k]$ and $y[k]$, respectively, and $\Gamma_{x y}(\nu)$ is the Fourier transform of the intercorrelation function $\gamma_{x y}[k]=E\left\{x[m] y^{*}[m-k]\right\}$. It is known that the matrix $\Gamma(\nu)$ is Hermitian and nonnegative definite (see [1, p. 235]).

We shall investigate the case where the rank of $\boldsymbol{\Gamma}(\nu)$ is one for any value of $\nu$. This means that the two columns are proportional or that there exists a complex function $G(\nu)$ such that

$$
\begin{aligned}
\Gamma_{x y}(\nu) & =G^{*}(\nu) \Gamma_{x}(\nu) \\
\Gamma_{y}(\nu) & =G^{*}(\nu) \Gamma_{y x}(\nu) .
\end{aligned}
$$


We deduce from (69a) and from the Hermitian property of $\boldsymbol{\Gamma}(\nu)$ that $\Gamma_{y x}(\nu)=G(\nu) \Gamma_{x}(\nu)$. Using this relation in (69b) yields $\Gamma_{y}(\nu)=|G(\nu)|^{2} \Gamma_{x}(\nu)$. Therefore, if the rank of $\boldsymbol{\Gamma}(\nu)$ is one, $\boldsymbol{\Gamma}(\nu)$ can expressed as

$$
\boldsymbol{\Gamma}(\nu)=\Gamma_{x}(\nu)\left[\begin{array}{cc}
1 & G^{*}(\nu) \\
G(\nu) & |G(\nu)|^{2}
\end{array}\right] .
$$

Let $\bar{y}[k]$ be the signal obtained by filtering $x[k]$ in the filter with frequency response $G(\nu)$. Using (69) and the classical expressions of linear filtering, we obtain

$$
\begin{aligned}
\Gamma_{\bar{y}}(\nu) & =|G(\nu)|^{2} \Gamma_{x}(\nu) \\
\Gamma_{y \bar{y}}(\nu) & =G^{*}(\nu) \Gamma_{y x}(\nu)=|G(\nu)|^{2} \Gamma_{x}(\nu) \\
\Gamma_{\bar{y} y}(\nu) & =G(\nu) \Gamma_{x y}(\nu)=|G(\nu)|^{2} \Gamma_{x}(\nu) .
\end{aligned}
$$

By Fourier transformation, we deduce that $E\{\mid y[k]-$ $\left.\left.\bar{y}[k]\right|^{2}\right\}=0$, which means that $y[k]=\bar{y}[k]$ with probability one. Therefore, if the rank of $\boldsymbol{\Gamma}(\nu)$ is one, the component $y[k]$ of the vector $[x[k], y[k]]^{T}$ is deduced from $x[k]$ by a linear filtering.

Conversely, if this property is satisfied, the spectral matrix takes the form (70), and therefore, its rank is one.

\section{APPENDIX B}

The assumptions on $w[k]$ are characterized by

$$
\begin{aligned}
E\left\{w[k] w^{*}[k-p]\right\} & =\delta[p], \\
E\{w[k] w[k-p]\} & =0 .
\end{aligned}
$$

Let us write (40) in the form $z[k]=a[k]+b[k]$, where $a[k]$ and $b[k]$ are deduced from $w[k]$ and $w^{*}[k]$ by linear filtering with frequency response $G(\nu)$ and $H(\nu)$, respectively. Then, it results from (72) that

$$
\begin{aligned}
\gamma_{z}[p] & =E\left\{z[k] z^{*}[k-p]\right\}=\gamma_{a}[p]+\gamma_{b}[p] \\
r_{z}[p] & =E\{z[k] z[k-p]\} \\
& =E\{a[k] b[k-p]\}+E\{b[k] a[k-p]\} .
\end{aligned}
$$

Using the definitions of $a[k]$ and $b[k]$ in (40), we obtain, by Fourier transformation,

$$
\begin{aligned}
& \Gamma_{z}(\nu)=|G(\nu)|^{2}+|H(\nu)|^{2} \\
& R_{z}(\nu)=G(\nu) H(-\nu)+G(-\nu) H(\nu) .
\end{aligned}
$$

\section{APPENDIX C}

The error $\epsilon_{L}^{2}$ obtained in linear prediction is given by $E\left(|z[k]|^{2}\right)-E\left(\left|a^{*} z[k-1]\right|^{2}\right)$. Noting that $|a|^{2}=\left|\gamma_{1}\right|^{2} / \gamma_{0}^{2}$, we obtain

$$
\epsilon_{L}^{2}=\gamma_{0}-\left(1 / \gamma_{0}\right)\left|\gamma_{1}\right|^{2}
$$

By replacing in (60) the value of $u$ deduced from (57), we deduce

$$
\epsilon_{W L}^{2}=\gamma_{0}-\left(1 / \gamma_{0}\right)\left|\gamma_{1}\right|^{2}-\left(1 / \gamma_{0}\right) v\left(\gamma_{0} r_{1}-\gamma_{1} r_{0}\right) .
$$

The content of the last brackets of this equation appears in (59) and is equal to $\Delta v^{*}$. Subtracting (78) from (77), we obtain (61).

\section{APPENDIX D}

Using the recursion $z[k]=a^{*} z[k-1]+w[k]$ and noting that $w[k]$ and $z[k-1]$ are uncorrelated, we obtain

$$
\gamma_{0}=|a|^{2} \gamma_{0}+\gamma_{w}
$$

which gives (62). It results from the same reasoning that $\gamma_{1}=a^{*} \gamma_{0}$. Now, the coefficient $r_{0}$ is given by

$$
r_{0}=E\left(z^{2}[k]\right)=E\left\{\left(a^{*} z[k-1]+w[k]\right)^{2}\right\} .
$$

Developing (80) yields

$$
r_{0}=a^{* 2} r_{0}+\gamma_{w} \rho_{0}+2 a^{*} \gamma_{w} T
$$

where

$$
\gamma_{w} T=E(w[k] z[k-1])
$$

The difference equation defining the AR(1) model introduces a causal linear filter with an impulse response equal to $a^{* k}$. We thus have

$$
z[k-1]=\sum_{l=0}^{\infty} a^{* k} w[k-1-l]
$$

and using this relation in (82) yields (64). Similarly, we have

$$
\begin{aligned}
r_{1} & =E(z[k] z[k-1]) \\
& =E\left\{\left(a^{*} z[k-1]+w[k]\right) z[k-1]\right\}
\end{aligned}
$$

from which (66) can be obtained.

\section{REFERENCES}

[1] B. Picinbono, Random Signals and Systems. Englewood Cliffs, NJ: Prentice-Hall, 1993.

[2] S. Kay, Modern Spectral Analysis; Theory and Applications. Englewood Cliffs, NJ: Prentice-Hall, 1988.

[3] B. Picinbono, "On circularity," IEEE Trans. Signal Processing, vol. 42, pp. 3473-3482, Dec. 1994.

[4] K. Miller, Complex Stochastic Processes, An Introduction to Theory and Application. Reading, MA: Addison-Wesley, 1974.

[5] P. Bondon and B. Picinbono, "De la blancheur et de ses transformations," Traitement du Signal, vol. 7, pp. 385-395, 1990.

[6] P. Bondon, P. L. Combettes, and B. Picinbono, "Volterra filtering and higher order whiteness," IEEE Trans. Signal Processing, vol. 43, pp. 2209-2212, Sept. 1995.

[7] J. L. Doob, Stochastic Processes. New York: Wiley, 1953.

[8] A. M. Yaglom, An Introduction to the Theory of Stationary Random Functions. New-York: Dover, 1962.

[9] H. Cramer and M. Leadbetter, Stationary and Related Stochastic Processes. New York: Wiley, 1967.

[10] E. Wong, Stochastic Processes in Information and Dynamical Systems New York: McGraw-Hill, 1971.

[11] M. Rosenblatt, Stationary Sequences and Random Fields. Boston, MA Birkhaüser, 1985.

[12] A. Blanc-Lapierre and R. Fortet, Théorie des Fonctions Aléatoires. Paris: Masson, 1953.

[13] K. Miller, Multidimensional Gaussian Distributions. New York: Wiley, 1964.

[14] M. Loève, Probabilty Theory. New York: Springer Verlag, 1978.

[15] B. Picinbono and P. Chevalier, "Widely linear systems for estimation," IEEE Trans. Signal Processing, vol. 43, pp. 2030-2033, Sept. 1995.

[16] _ "Extensions of the minimum variance method," Signal Processing, vol. 49, pp. 1-9, 1996. 


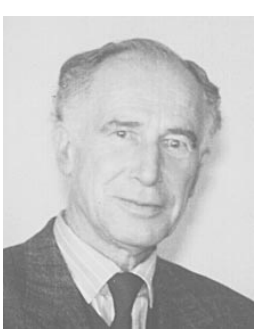

Bernard Picinbono (M'63-SM'79-F'85) was born in Algiers, Algeria, on March 21, 1933. He received the Agrégation des Sciences Physiques degree in 1956 and the Doctorat d'état degree in 1960.

From 1960 to 1965, he was a Professor at the University of Algiers, Algeria, and since 1965, he has been with the Department of Physics of the University of Paris-Sud, Orsay, France. He was the President of this university from 1970 to 1975.

He was Director of the Laboratoire des Signaux et Systèmes, a joint laboratory of the Centre National de la Recherche Scientifique (CNRS), the École Supérieure d'Électricité (Supélec) and the University of Paris-Sud between 1975 and 1987. From 1990 to 1995, he was General Director of Supélec. His research interests include statistical signal processing, detection and estimation, and stochastic processes. He is the author of numerous papers and books on these subjects.

Dr. Picinbono is a member of the French Academy of Sciences and President of the GRETSI, with which he organizes, every two years in France, a conference that is mainly devoted to signal and image processing.

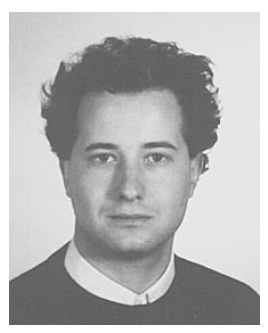

Pascal Bondon (S'91-M'93) was born in Paris, France, in 1964. He received the Agrégation de Sciences Physiques degree from the École Normale Supérieure, Cachan, France, in 1987 and the Doctorat en Sciences from the University of Paris-Sud, Orsay, France, in 1991.

From 1988 to 1992 , he was a teaching and research assistant at the University of Paris-Sud. Since 1992, he has been Chargé de Recherche at the CNRS, Laboratoire des Signaux et Systèmes CNRS (UMR 14), Gif-sur-Yvette, France, which is a joint research center of the CNRS, the École Supérieure d'Électricité, and the University of Paris-Sud. His general interests include signal processing, nonlinear time series analysis, and estimation theory. 